\title{
Disorder effects in epitaxial thin films of $(\mathrm{La}, \mathrm{Ca}) \mathrm{MnO}_{3}$
}

J. Aarts, S. Freisem, and R. HendrikxH. W. Zandbergen

Citation: 72, (1998); doi: 10.1063/1.121512

View online: http://dx.doi.org/10.1063/1.121512

View Table of Contents: http://aip.scitation.org/toc/apl/72/23

Published by the American Institute of Physics 


\title{
Disorder effects in epitaxial thin films of $(\mathrm{La}, \mathrm{Ca}) \mathrm{MnO}_{3}$
}

\author{
J. Aarts, ${ }^{\text {a) }}$ S. Freisem, and R. Hendrikx \\ Kamerlingh Onnes Laboratory, Leiden University, 2300 RA Leiden, the Netherlands \\ H. W. Zandbergen \\ Material Science Department, Technical University Delft, the Netherlands
}

(Received 3 March 1998; accepted for publication 3 April 1998)

\begin{abstract}
We have investigated as-grown sputtered films of $\mathrm{La}_{0.7} \mathrm{Ca}_{0.3} \mathrm{MnO}_{3}$ in a thickness range between 5 and $200 \mathrm{~nm}$ on $\mathrm{SrTiO}_{3}$ substrates. The films are epitaxial, strained, and smooth. All films order magnetically around $175 \mathrm{~K}$. Very thin films show full magnetization at low temperatures, but the temperature of the metal-insulator transition is appreciably lower than the magnetic ordering temperature. In thick films, the magnetization is much lower than expected. Both effects are probably related to structural disorder as found by transmission electron microscopy. (C) 1998 American Institute of Physics. [S0003-6951(98)01123-1]
\end{abstract}

The observation of "colossal" magnetoresistance (CMR) effects in thin films of manganite perovskites ${ }^{1}$ has led to a number of investigations into their transport and magnetic properties, which were found to depend strongly on sample morphology: single crystals, polycrystals, powders, and films all have different magnetoresistance (MR) behavior. Focusing on $\mathrm{La}_{0.7} \mathrm{Ca}_{0.3} \mathrm{MnO}_{3}$ (LCMO), in the metallic doping regime, measurements on annealed powders show a Curie temperature $T_{c}$ of about $250 \mathrm{~K}$, a peak temperature $T_{p}$ of the resistance just below $T_{c}$, and only a moderate magnetoresistance ratio (MRR) of about 5 in $4 \mathrm{~T}^{2}$ These numbers can be widely different for films, due to the extreme sensitivity of the physical properties of the material to structural changes or disorder. Consequently, deposition parameters, oxygen content, but also lattice strain due to the underlying substrate, all can have an influence. Disorder and strain effects can also be influenced by postanneal procedures. Hundley $e t a l .^{3}$ showed that with proper annealing at high temperature $\left(950{ }^{\circ} \mathrm{C}\right)$ and oxygen pressure $\left(10^{5} \mathrm{~Pa}\right)$, the values for $T_{c}, T_{p}$, and the MRR can become equal to the powder values quoted above. The precise morphology changes induced by the annealing are not exactly known. Among them is most probably strain relief, since the film surface usually changes from atomically smooth to rough on a scale of several $\mathrm{nm},{ }^{3}$ which would be due to the formation of relaxed grains. However, postannealing is often undesirable if the goal is to combine films with different physical properties (e.g., CMR and high- $T_{c}$ materials), nor is it a prerequisite for producing epitaxial and smooth films. ${ }^{4,5}$ On the other hand, such epitaxial films usually show lower $T_{c}$ 's and spreads in $T_{p}$ values. Understanding the differences between films and bulk material is necessary both for applications involving heteroepitaxy (e.g., spin devices or tunnel junctions), and for elucidating the link between the microstructure and MR behavior. Especially very thin films (in the range of $10 \mathrm{~nm}$ ) deserve attention, since they can be expected to be more uniformly strained and oxidized.

In this letter we study transport, magnetization, and film

${ }^{a)}$ Electronic mail: aarts@rulkol.leidenuniv.nl structure for films of thickness $d_{s}$ ranging from 5 to $200 \mathrm{~nm}$, deposited by high-temperature oxygen sputtering on $\mathrm{SrTiO}_{3}$ (STO) substrates at an elevated temperature. The films are epitaxial and smooth at all thicknesses, but we find major changes in their properties. First, $T_{c}$ is constant around 175 $\mathrm{K}$ for all $d_{s}$, and $T_{p}$ lies just below $T_{c}$ for most thicknesses, but below $30 \mathrm{~nm}$ this connection is lost and $T_{p}$ decreases strongly. Second, the high-field magnetization $M_{f}$ has the expected value of about $3.5 \mu_{B} / \mathrm{Mn}$ for small $d_{s}$ but decreases strongly with increasing thickness. Both effects point to the presence of different kinds of disorder, for which possible sources are identified by high-resolution electron microscopy (HREM).

All films were sputter deposited from ceramic targets of $\mathrm{La}_{1-x} \mathrm{Ca}_{x} \mathrm{MnO}_{3}$ with a nominal composition of $x=0.33$ on STO substrates, in a pure oxygen atmosphere of $300 \mathrm{~Pa}$, with substrate and source in line and about $2 \mathrm{~cm}$ apart. The high pressure causes a very low growth rate of the order of 0.9 $\mathrm{nm} / \mathrm{min}$. The growth temperature was chosen at $840{ }^{\circ} \mathrm{C}$, in order to be able to grow high-quality films of $\mathrm{YBa}_{2} \mathrm{Cu}_{3} \mathrm{O}_{7}$ under the same conditions. After deposition, each sample was cooled to room temperature without further annealing. The chemical composition of the films was determined by microprobe analysis, which showed a $\mathrm{Ca}$ content of $x$ $=0.27$. The crystal structure was determined by $x$-ray diffraction and HREM; transport measurements were performed on unpatterned samples with sputtered gold contacts; and the magnetization was measured with a superconducting quantum interference device magnetometer.

Figure 1 shows typical data of resistance $R$ and magnetization $M$ in $0.3 \mathrm{~T}$ as a function of temperature $T$ for $d_{s}$ $=23$ and $57 \mathrm{~nm}$. As indicated, the peak in $R$ defines $T_{p}$, while the intercept of the linearly increasing $M(T)$ with the constant magnetization at high temperatures is used to determine $T_{c}$. Note that $M(T)$ for $d_{s}=23 \mathrm{~nm}$ is larger than for 57 $\mathrm{nm}$, and that for the thinner film $T_{p}$ is much lower than $T_{c}$. Figure 2 shows the magnetization $M_{f}$ of a typical series of films with $d_{s}$ between 5 and $100 \mathrm{~nm}$ as a function of magnetic-field $B_{a}$ at $5 \mathrm{~K}$. Values for $M_{f}$ were obtained by correcting the measured magnetization for the contribution of the substrate, using a susceptibility value of -1.3 


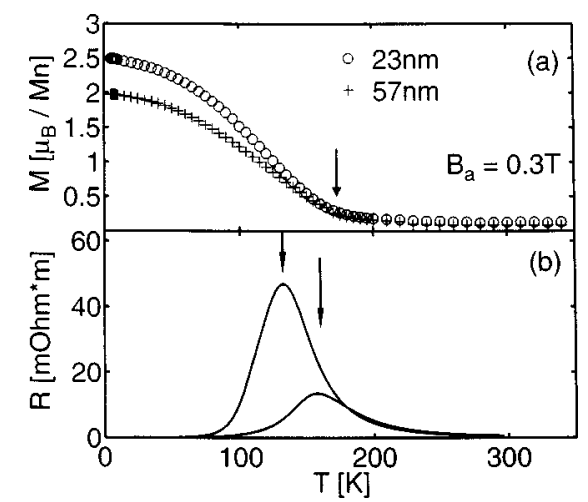

FIG. 1. (a) Magnetization $M$ as a function of temperature $T$ for films of 23 $\mathrm{nm}(\bigcirc)$ and $57 \mathrm{~nm}(+)$ in an applied field of $0.3 \mathrm{~T}$. The arrow denotes the Curie temperatures. (b) Resistance $R$ vs $T$ of the same films. The arrows indicate peak temperatures $T_{p}$.

$\times 10^{-9} \mathrm{~m}^{3} / \mathrm{kg}$, as measured on a bare substrate. In Fig. 3, different quantities are collected as a function of $d_{s}$. Figure 3 (a) shows the lattice parameters $b_{p}$ along the $b$ axis, defined to be perpendicular to the substrate. They were determined from the (002) peak in a $\vartheta-2 \vartheta$ scan (indexed on a pseudocubic unit cell), compared to the equivalent STO peak with a lattice parameter of $0.3905 \mathrm{~nm}$. For all samples, rocking curve widths $\left(\vartheta-\vartheta\right.$ scan) were found to be below $0.07^{\circ}$. The values for $b_{p} \approx 0.383 \mathrm{~nm}$ are significantly smaller than the bulk value of $0.386 \mathrm{~nm}$. This tetragonal contraction of the unit cell, present at all $d_{s}$, is probably due to strain in the film plane, induced by epitaxial growth on the larger substrate. Strain, but also pressure, should have an effect on $T_{c}$, which is plotted in Fig. 3(b). The value of $T_{c} \approx 175 \mathrm{~K}$ is much lower than the bulk value of $250 \mathrm{~K}$, which can be either due to strain, or to the oxygen content. In polycrystalline $\mathrm{La}_{0.67} \mathrm{Ba}_{0.33} \mathrm{MnO}_{3-\delta}$, decreasing $\delta$ led to serious lowering of $T_{c}\left(\Delta T_{c} \approx-70 \mathrm{~K}\right.$ from $\delta=0.01$ to $\left.\delta=0.09\right) .{ }^{6}$ In our films this is probably not the case. A short (15 min) postanneal at $950{ }^{\circ} \mathrm{C}$ in flowing oxygen did not show an appreciable change in $T_{c}$, although the time should be long enough to reach oxygen equilibrium. ${ }^{7}$ With respect to strain effects, it should be remarked that the unit cell volume is not conserved. Assuming an in-plane lattice parameter of 0.3905 $\mathrm{nm}$, the volume is $5.84 \times 10^{-2}(\mathrm{~nm})^{3}$, compared to 5.75 $\times 10^{-2}(\mathrm{~nm})^{3}$ for the bulk unit cell. The (negative) equivalent hydrostatic pressure for this volume change can be esti-

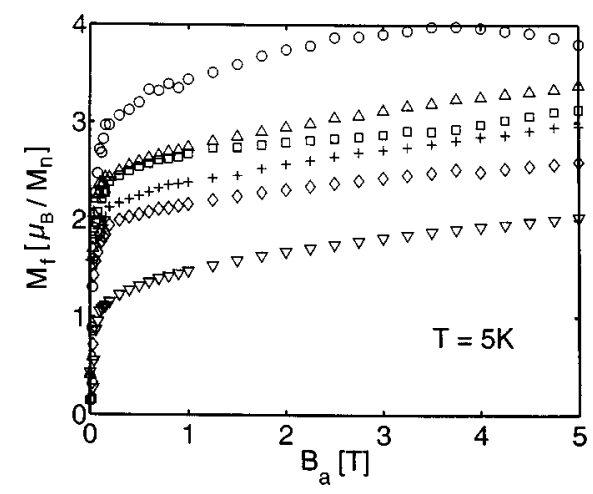

FIG. 2. Magnetization $M_{f}$ as a function of applied magnetic-field $B_{a}$ at $5 \mathrm{~K}$, I the $19 \mathrm{~nm}$ film, $M_{f}$ appears somewhat too low.

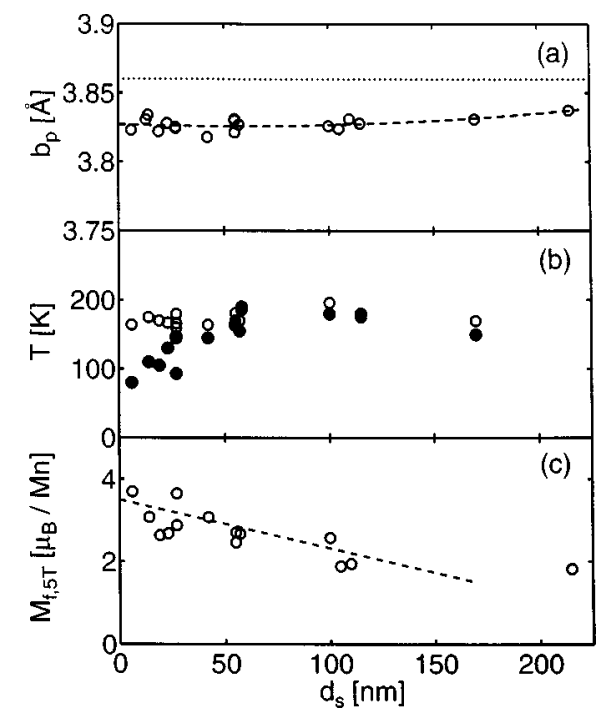

FIG. 3. (a) Length of the pseudocubic $b$-axis $b_{p}$ as a function of film thickness $d_{s}$. The dotted line indicates the bulk value; the dashed line is meant to guide the eye. (b) Values for Curie temperature $T_{c}(\bigcirc)$ and resistance peak temperature $T_{p}$ as a function of $d_{s}$. (c) Magnetization $M_{f, 5 \mathrm{~T}}$ in 5 $\mathrm{T}$ at $5 \mathrm{~K}$ as a function of $d_{s}$. The dashed line is meant to guide the eye.

mated at $5 \mathrm{GPa},{ }^{8}$ which could lead to $\Delta T_{c} \simeq-20 \mathrm{~K},{ }^{9}$ too small to explain the experimental value. However, biaxial strain can have an equal or even larger effect on $T_{c}$, as was recently argued by Millis et al. ${ }^{10} \mathrm{We}$ believe, therefore, that our measured values are intrinsic for epitaxial, strained LCMO on STO, which is corroborated by similar values reported previously. ${ }^{4,5,11}$ Note that growth on LAO indicates different behavior, with strained, epitaxial films yielding $T_{c}$ values around $240 \mathrm{~K}^{11}$

Next, we turn to the behavior of the films with small $d_{s}$. Figure 3(b) shows the values for $T_{p}$ as well as for $T_{c}$, while Fig. 3(c) shows the values of $M_{f}$ at $5 \mathrm{~K}$ in $5 \mathrm{~T}$. Below about $30 \mathrm{~nm} T_{p}$ starts to decrease, while $T_{c}$ remains constant and $M_{f}$ is about $3.5 \mu_{B} / \mathrm{Mn}$. In other words, magnetically the films behave as expected, but a decoupling takes place between the magnetic transition and the metal-insulator transition. Apparently, the disorder in the films is too strong at $T_{c}$ to allow the formation of a metallic state, but this is overcome at lower temperature by the growing average magnetization, which favors metallic conductivity. The data also provide some coarse estimate for typical localization lengths in these films. They must be clearly larger than interatomic distances, since the ferromagnetism is due to electrons hopping with spin memory, but smaller than the film thickness of $30 \mathrm{~nm}$ (75 unit cells) at which $T_{p}$ starts to decrease.

On increasing $d_{s}$, different behavior occurs: $T_{p}$ is now close to $T_{c}$, but $M_{f}$ gradually decreases from values around $3.5 \mu_{B} / \mathrm{Mn}$ for the thin films to less than $1.5 \mu_{B} / \mathrm{Mn}$ at 215 $\mathrm{nm}$. Again, this must be due to some kind of disorder, which causes increasing amounts of Mn moments to be antiferromagnetically coupled. We know of no similar findings; the magnetization has not been investigated systematically, although too low values for films around $100 \mathrm{~nm}$ are reported routinely. ${ }^{12-14}$ Of course, this must have serious consequences for the transport currents. As they will not flow in antiferromagnetic regions, the current distribution in these films is probably inhomogeneous. 


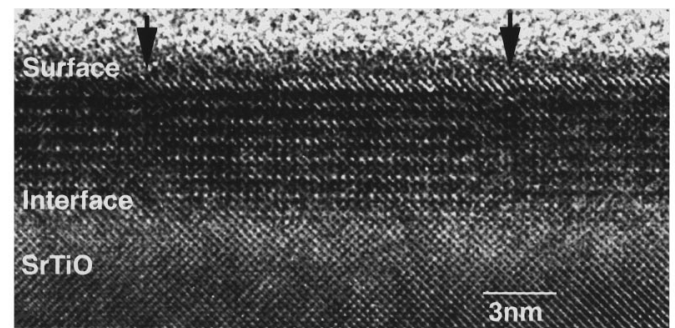

(a)

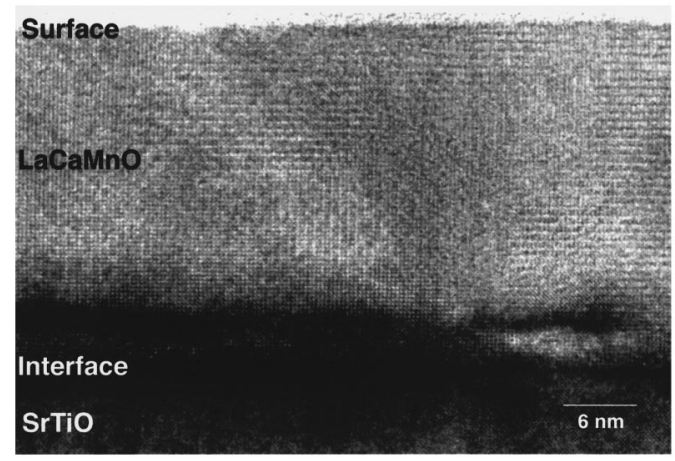

(b)

FIG. 4. HREM images for two films with different thickness $d_{s}$. (a) $d_{s}$ $=5 \mathrm{~nm}$. The specimen orientation is about $2^{\circ}$ from the [100] zone axis of $\mathrm{SrTiO}_{3}$ towards the [011] $\mathrm{SrTiO}_{3}$ direction, to allow imaging of the LCMO (010) fringe. Two antiphase boundaries are visible, marked with arrows. (b) $d_{s}=30 \mathrm{~nm}$. Two domain directions are clearly visible. The lower-left-hand corner shows a cubic-like structure with the $b$ axis in the line of sight. The upper-middle and right-hand parts show structures with larger spacing where the $b$ axis is perpendicular to the interface.

Having found different signatures for disorder, a closer look at the atomic structure is needed. Shown in Fig. 4 are two HREM images of films of 5 and $30 \mathrm{~nm}$; a thick film (215 $\mathrm{nm})$ was also investigated. Common to all three films is that they are epitaxial; that only few dislocations could be found, and certainly less than would be expected on the basis of a rigid body fitting of the atomic lattices of film and substrate; and that the lattice deformation imposed by the substrate persists up to the film surface. These observations confirm the conclusions from x-ray diffraction that the films are homogeneously strained and epitaxial. However, the images contain more information. In films, the crystal structure is usually referred to as pseudocubic, characterized by a single lattice parameter $a_{p}$; small rotations of the oxygen octahedra actually lead to an orthorhombic unit cell of dimensions $\sqrt{2} a_{p}, 2 a_{p}, \sqrt{2} a_{p} .{ }^{15}$ This superstructure is found everywhere in the images, but there are qualitative differences between the films of 5 and $30 \mathrm{~nm}$. In the $5 \mathrm{~nm}$ film, the $b$ axis is always perpendicular to the interface. This is reasonable, since it is the direction of the largest mismatch. However, defects are visible in the form of antiphase boundaries (APB's), where the $b$-axis periodicity shifts over $a_{p}$ (half its length). The typical APB distance is around $10 \mathrm{~nm}$. In the 30 $\mathrm{nm}$ film, no APB's are found, but three different types of regions occur, with the three possible directions of the $b$ axis. The typical size of these regions ranges from a third of $d_{s}$ to three times $d_{s}$, in the $30 \mathrm{~nm}$ film as well as in the 215 nm film.

In short, the microstructure of films with a thickness below $30 \mathrm{~nm}$ is different from the microstructure found for thicker films, which offers an explanation for the differences in the behavior of $T_{c}$ (constant) and $T_{p}$ (decreasing). For the thicker films, the most noteworthy point is the domain type of disorder. These domains are not necessarily formed during growth. Distorted (orthorhombic) perovskites usually undergo a phase transition to a more symmetric structure at elevated temperature. For bulk LCMO, differential thermal analysis data indicate a phase transition around $500{ }^{\circ} \mathrm{C},{ }^{16}$ which makes it likely that, also in films, the structure at the growth temperature is different from the one at low temperatures. In that case, the domains could be formed during cooling of the sample. Not clear at this point is whether the domain-type disorder is responsible for the loss of magnetic moment.

The authors thank A. J. Millis, L. F. Cohen, J. A. Mydosh, and P. H. Kes for discussions. This work is part of the research program of the "Stichting voor Fundamenteel Onderzoek der Materie" (FOM), which is financially supported by NWO.

${ }^{1}$ R. v. Helmholt, J. Wecker, B. Holzapfel, L. Schultz, and K. Samwer, Phys. Rev. Lett. 71, 2331 (1993).

${ }^{2}$ P. Schiffer, A. P. Ramirez, W. Bao, and S.-W. Cheong, Phys. Rev. Lett. 75, 3336 (1995)

${ }^{3}$ M. F. Hundley, M. Hawley, R. H. Heffner, Q. X. Jia, J. J. Neumeier, J. Tesmer, J. D. Thompson, and X. D. Wu, Appl. Phys. Lett. 67, 860 (1995).

${ }^{4}$ V. A. Vas'ko, C. A. Nordman, P. A. Kraus, V. S. Achutaraman, A. R. Ruosi, and A. M. Goldman, Appl. Phys. Lett. 68, 2571 (1996).

${ }^{5}$ J. N. Eckstein, I. Bozovic, J. O'Donnell, M. Onellion, and M. S. Rzchowsky, Appl. Phys. Lett. 69, 1312 (1996).

${ }^{6}$ H. L. Ju, J. Gopalakrishnan, J. L. Peng, Qi Li, G. C. Xiong, T. Venkatesan, and R. L. Greene, Appl. Phys. Lett. 51, 6143 (1995).

${ }^{7}$ K. A. Thomas, P. S. I. P. N. de Silva, L. F. Cohen, A. Hossain, M. Rajeswan, T. Venkatesan, R. Hiskes, and J. L. MacManus-Driscoll (unpublished).

${ }^{8}$ H. Y. Hwang, T. T. M. Palstra, S.-W. Cheong, and B. Batlogg, Phys. Rev. B 52, 15046 (1995), quoting an average pressure effect of 4 $\times 10^{-4} / \mathrm{kbar}$

${ }^{9}$ Y. Moritomo, A. Asamitsu, and Y. Tokura, Phys. Rev. B 51, 16491 (1995), measured on $\mathrm{La}_{0.7} \mathrm{Sr}_{0.3} \mathrm{MnO}_{3}$.

${ }^{10}$ A. J. Millis, T. Darling, and A. Migliori, J. Appl. Phys. 83, 1588 (1998).

${ }^{11}$ T. Y. Koo, S. H. Park, and Y. H. Jeong, Appl. Phys. Lett. 71, 977 (1997).

${ }^{12}$ S. Jin, T. H. Tiefel, M. McCormack, R. A. Fastnacht, R. Ramesh, and L. H. Chen, Science 264, 413 (1994).

${ }^{13}$ C. L. Canedy, K. B. Ibsen, G. Xiao, J. Z. Sun, A. Gupta, and W. J. Gallagher, Appl. Phys. Lett. 79, 4546 (1996).

${ }^{14}$ A. Gupta, G. Q. Gong, G. Xiao, P. R. Duncombe, P. Lecoeur, P. Trouilloud, Y. Y. Wang, V. P. Dravid, and J. Z. Sun, Phys. Rev. B 54, R15 629 (1996); see also S. Jin et al., Appl. Phys. Lett. 67, 557 (1995); J. Q. Guo et al., J. Appl. Phys. 81, 7445 (1997).

${ }^{15}$ P. Dai, J. Zhang, H. A. Mook, S.-H. Liou, P. A. Douben, and E. W. Plummer, Phys. Rev. B 54, R3694 (1996).

${ }^{16}$ T.-W. Li (private communication). 